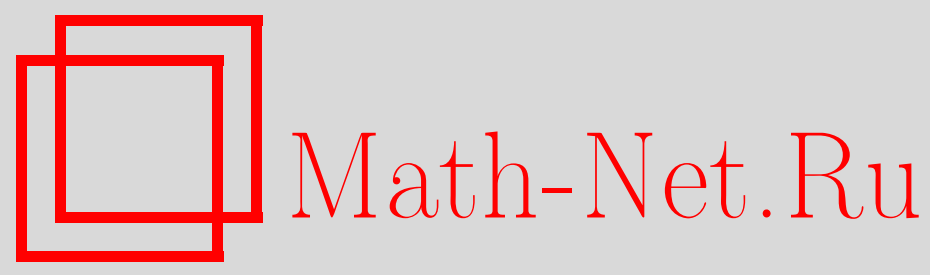

Е. Д. Лившиц, О скорости сходимости чисто жадного алгоритма, Матем. заметки, 2004, том 76, выпуск 4, 539-552

DOI: https://doi.org/10.4213/mzm130

Использование Общероссийского математического портала Math-Net.Ru подразумевает, что вы прочитали и согласны с пользовательским соглашением http://www. mathnet.ru/rus/agreement

Параметры загрузки:

IP : 54.89 .56 .158

26 апреля 2023 г., 10:25:47

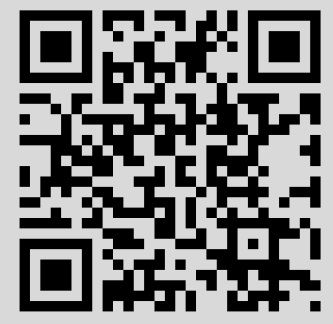


УДК 517.518 .8

\title{
О СКОРОСТИ СХОДИМОСТИ ЧИСТО ЖАДНОГО АЛГОРИТМА
}

\section{Е. Д. Лившиц}

\begin{abstract}
Статья посвящена изучению скорости сходимости чисто жадного алгоритма (гриди-алгоритма) в гильбертовом пространстве. Получены оценки сверху на скорость сходимости чисто жадного алгоритма для функций из класса $A_{\alpha, \beta}(D)$.

Библиографояи: 4 названия.
\end{abstract}

1. Введение. Настоящая работа посвящена исследованию скорости сходимости чисто жадного алгоритма (гриди-алгоритма) в действительном гильбертовом пространстве $H$. Для определения чисто жадного алгоритма в пространстве $H$ необходим словарь $\mathscr{D}$ - нормированное подмножество $H$, линейная оболочка которого всюду плотна, и начальньй вектор $f_{0} \in H$.

Для произвольного $f \in H$ выберем такой вектор $g(f) \in \mathscr{D}$, который максимизирует $|\langle f, g\rangle|$ (мы будем полагать, что он существует). Определим

$$
G(f, \mathscr{D}):=\langle f, g(f)\rangle g(f) \quad \text { и } \quad R(f, \mathscr{D}):=f-G(f, \mathscr{D}) .
$$

ЧИСТо ЖАДНЫЙ АлГОритм (ЧЖА). Положим $R_{0}\left(f_{0}, \mathscr{D}\right):=f_{0}$ и $G_{0}\left(f_{0}, \mathscr{D}\right):=0$. Тогда для каждого $m \geqslant 0$ последовательно определяем

$$
\begin{gathered}
g_{m+1}:=g\left(f_{m}\right), \\
G_{m+1}\left(f_{0}, \mathscr{D}\right):=G_{m}\left(f_{0}, \mathscr{D}\right)+G\left(R_{m}\left(f_{0}, \mathscr{D}\right), \mathscr{D}\right)=G_{m}\left(f_{0}, \mathscr{D}\right)+\left\langle f_{m}, g_{m+1}\right\rangle g_{m+1} \\
R_{m+1}\left(f_{0}, \mathscr{D}\right):=f_{m+1}:=f-G_{m+1}\left(f_{0}, \mathscr{D}\right)=R\left(R_{m}\left(f_{0}, \mathscr{D}\right), \mathscr{D}\right)=f_{m}-\left\langle f_{m}, g_{m+1}\right\rangle g_{m+1}
\end{gathered}
$$

Формальньй ряд

$$
\sum_{m=0}^{\infty}\left\langle f_{m}, g_{m+1}\right\rangle g_{m+1}
$$

также назьвают PGA-разложсением вектора $f_{0}$ по словарю $\mathscr{D}$.

Джонс [1] доказал, что ЧЖА в гильбертовом пространстве сходится, т.е.

$$
G_{m}\left(f_{0}, \mathscr{D}\right) \rightarrow f_{0}, \quad f_{m}=R_{m}\left(f_{0}, \mathscr{D}\right) \rightarrow 0 \quad \text { при } m \rightarrow \infty
$$

Работа выполнена при финансовой поддержке Российского фонда фундаментальных исследований, грант № 02-01-00248. 
для всех словарей $\mathscr{D}$ и начальных векторов $f_{0}$.

Ясно, что нельзя дать общих оценок на скорость сходимости ЧЖА (PGA-разложений) для произвольного $D$ и произвольного $f_{0}$. Поэтому обычно ставится вопрос о получении оценок на скорость сходимости ЧЖА в случае, если начальньй вектор $f_{0}$ принадлежит некоторому классу, зависящему от $\mathscr{D}$. Во многих работах по жадным алгоритмам в качестве такого класса рассматривается класс $\mathscr{A}_{1}(\mathscr{D})$ - замькание выпуклой оболочки векторов, пропорциональных векторам из словаря $\mathscr{D}$.

Более точно, сначала определяется класс функций

$$
\mathscr{A}_{1}^{o}(\mathscr{D}, M):=\left\{f \in H: f=\sum_{k \in \Lambda} c_{k} w_{k}, w_{k} \in \mathscr{D}, \# \Lambda<\infty \text { и } \sum_{k \in \Lambda}\left|c_{k}\right| \leqslant M\right\}
$$

и класс $\mathscr{A}_{1}(\mathscr{D}, M)$, являющийся замыканием (в $\left.H\right)$ класса $\mathscr{A}_{1}^{o}(\mathscr{D}, M)$. Далее, определим $\mathscr{A}_{1}(\mathscr{D})$ как объединение по $M>0$ всех $\mathscr{A}_{1}(\mathscr{D}, M)$. Для $f \in \mathscr{A}_{1}(\mathscr{D})$ введем норму

$$
|f|_{\mathscr{A}_{1}(\mathscr{D})}
$$

как наименьшее $M$, для которого $f \in \mathscr{A}_{1}(\mathscr{D}, M)$.

В работе [2] Девором и Темляковым была получена первая оценка сверху на скорость сходимости ЧЖА для функций из класса $\mathscr{A}_{1}(\mathscr{D})$ :

$$
\left\|f-G_{m}(f, \mathscr{D})\right\| \leqslant|f|_{\mathscr{A}_{1}(\mathscr{D})} m^{-1 / 6}
$$

Это оценка была улучшена Конягиньм и Темляковым в [3]. Они доказали, что

$$
\left\|f-G_{m}(f, \mathscr{D})\right\| \leqslant 4|f|_{\mathscr{A}_{1}(\mathscr{D})} m^{-11 / 62} .
$$

Оценка снизу на скорость сходимости ЧЖА в этом классе была получена Лившищем и Темляковьм в [4]. Было доказано существование словаря $\mathscr{D}$ и вектора $f \in H, f \neq 0$, для которых

$$
\left\|f-G_{m}(f, \mathscr{D})\right\| \geqslant C m^{-0.27}|f|_{\mathscr{A}_{1}(\mathscr{D})} .
$$

Возникает вопрос о скорости сходимости ЧЖА на других классах функций. Представляется естественным сравнить качество приближения, полученного после $m$ шагов чЖА, величину $\left\|f-G_{m}(f, \mathscr{D})\right\|$, с величиной наилучшего $m$-членного приближения $\sigma_{m}(f, \mathscr{D})$

$$
\sigma_{m}(f, \mathscr{D})=\inf _{\substack{c_{1}, \ldots, c_{m} \in \mathbb{R} \\ g_{1}, \ldots, g_{m} \in \mathscr{D}}}\left\|f-\sum_{k=1}^{m} c_{k} g_{k}\right\|
$$

Для $\gamma>0$ рассмотрим классы, состоящие из функций с фиксированной скоростью убывания $m$-членных приближений

$$
A^{\gamma}(\mathscr{D})=\left\{f \in H: \exists M \forall m \sigma_{m}(f, \mathscr{D}) \leqslant M m^{\gamma}\right\}
$$

Выяснилось, что в случае произвольного словаря $\mathscr{D}$ принадлежность функции $f$ классу $A^{\gamma}(\mathscr{D})$ не позволяет получить какую-либо степенную оценку сверху на скорость убывания $\left\|f-G_{m}(f, \mathscr{D})\right\|$, что иллюстрирует следующий 
ПримеР 1. Для произвольных $\gamma, \delta>0$ существует словарь $\mathscr{D}$, функция $f_{0} \in A^{\gamma}(\mathscr{D})$ и $C>0$ такие, что для всех $m>1$ вьполняется неравенство

$$
\left\|f-G_{m}(f, \mathscr{D})\right\| \geqslant C m^{-\delta} .
$$

(Соответствующее построение приведено в п. 4.)

Поэтому для получения содержательных оценок сверху необходимо ввести какиелибо дополнительные ограничения на функциональньй класс. В этой работе мы накладьваем не только условия на величину $m$-членного приближения, но и условие на $\mathscr{A}_{1}(\mathscr{D})$-норму $\left(|\cdot|_{\mathscr{A}_{1}(\mathscr{D})}\right)$ аппроксиманта. А именно, в качестве класса начальных функций рассматривается класс $A_{\alpha, \beta}(\mathscr{D})$, определенньй так:

$$
A_{\alpha, \beta}(\mathscr{D})=\bigcup_{M_{\alpha}, M_{\beta}>1} A_{\alpha, \beta}^{M_{\alpha}, M_{\beta}}(\mathscr{D})
$$

где

$$
\begin{gathered}
A_{\alpha, \beta}^{M_{\alpha}, M_{\beta}}(\mathscr{D})=\left\{f \in H \mid \forall m \exists g_{0}, \ldots, g_{m-1} \in \mathscr{D}, c_{0}, \ldots, c_{m-1} \in \mathbb{R}:\right. \\
\left.\left\|f-\sum_{i=0}^{m-1} c_{i} g_{i}\right\| \leqslant \frac{M_{\alpha}}{m^{\alpha}}, \sum_{i=0}^{m-1}\left|c_{i}\right| \leqslant M_{\beta} m^{\beta}\right\} .
\end{gathered}
$$

Для него получены следующие оценки на скорость сходимости.

Tеорема 1. Пусть $0<\alpha<1 / 6, \beta \geqslant 0, \alpha+\beta<1 / 2 u f \in A_{\alpha, \beta}(H, D)$. Тогда для любого $\varepsilon>0$ существует такое $C>0$, что для всех $m \geqslant 0$ выполняется

$$
\left\|f-G_{m}(f, D)\right\| \leqslant C m^{-\alpha /(1+2 \alpha)+\varepsilon} .
$$

Tеорема 2. Пусть $0<\alpha<1 / 6,0<\beta<1 / 2, \alpha /(1+2 \alpha)+\beta \geqslant 1 / 2 u f \in A_{\alpha, \beta}(H, D)$. Тогда для любого $\varepsilon>0$ существует такое $C>0$, ито для всех $m \geqslant 0$

$$
\left\|f-G_{m}(f, D)\right\| \leqslant C m^{-(1 / 2-\beta)+\varepsilon} .
$$

Объединяя теоремы 1 и 2, получаем следующую теорему.

Tеорема 3. Пусть $0<\alpha<1 / 6,0<\beta<1 / 2 u f \in A_{\alpha, \beta}(H, D)$. Тогда для любого $\varepsilon>0$ существует такое $C>0$, что для всех $m \geqslant 0$

$$
\left\|f-G_{m}(f, D)\right\| \leqslant C m^{-\min (1 / 2-\beta, \alpha /(1+2 \alpha))+\varepsilon} .
$$

Отметим, что при $\beta=0$ теорема 1 гарантирует скорость сходимости ЧЖА $C m^{-1 / 8+\varepsilon}$, что уступает оценке $(1.3)$, которая дает скорость $C m^{-11 / 62}$. С другой стороны, показатель в оценке $(1.4)$ не зависит от $\beta$, а классы $A_{\alpha, \beta}(\mathscr{D})$ расширяются с увеличением $\beta$; отсюда вытекает, что теоремы 1 и 3 "наиболее точны" в случае $\beta>1 / 2-\alpha-\varepsilon$. 
ЗАмЕчАниЕ. Как отмечалось вьше, элемент $g(f)$, максимизирующий $|\langle f, g\rangle|$ может не существовать. Чтобы избежать этой проблемы, можно выбирать в качестве $g(f)$ произвольный элемент словаря, удовлетворяющий неравенству

$$
|\langle f, g(f)\rangle| \geqslant t \sup _{g \in \mathscr{D}}|\langle f, g\rangle|,
$$

где $t, 0<t<1,-$ фиксированное число (что является простейшей разновидностью слабого жадного алгортима). В этом случае по наперед заданному $\varepsilon$ можно определить такое достаточно близкое к единице число $t$, что для соотвествующего алгоритма утверждения теорем 1-3 останутся истинными.

2. Доказательство теоремы 1. Ясно, что теорема 1 вытекает из следующего утверждения: для любого $\varepsilon_{0}>0$ существует $\delta>0$ такое, что для всех функций $f \in$ $A_{\alpha, \beta}^{1,1}(\mathscr{D}),\|f\|<\delta<1$, вьполняется

$$
\left\|f-G_{m}(f, D)\right\| \leqslant m^{-\alpha /(1+2 \alpha)+\varepsilon_{0}},
$$

которое мы и будем доказывать.

Пусть проводится ЧЖА по системе $\mathscr{D}$ с начальньм вектором $f_{0}:=f$, т.е. по формулам (1.1) и (1.2) определяются $f_{m}$ и $g_{m}$. Введем стандартные обозначения:

$$
a_{m}=\left\langle f_{m}, f_{m}\right\rangle, \quad d_{m}=\left|\left\langle f_{m}, g_{m+1}\right\rangle\right|, \quad b_{m+1}=\sum_{k=0}^{m} d_{m} .
$$

Из (1.2) следует равенство

$$
f_{m}=f_{0}-G_{m}\left(f_{0}, \mathscr{D}\right)=f_{0}-\sum_{j=0}^{m-1}\left\langle f_{j}, g_{j+1}\right\rangle g_{j+1}
$$

$\mathrm{C}$ другой стороны, функция $f_{0} \in A_{\alpha, \beta}^{1,1}$. Поэтому для всех $m$ найдутся такие $c_{k_{j}} \in \mathbb{R}$, $\widetilde{g}_{k_{j}} \in D, j=0, \ldots, m-1$ и $r_{m} \in H$, что

$$
f_{0}-\sum_{j=0}^{m-1} c_{k_{j}} \widetilde{g}_{k_{j}}=r_{m}, \quad\left\|r_{m}\right\| \leqslant m^{-\alpha}, \quad \sum_{j=0}^{m-1}\left|c_{k_{j}}\right| \leqslant m^{\beta} .
$$

Тогда

$$
\begin{aligned}
& a_{m}=\left\langle f_{m}, f_{m}\right\rangle=\left\langle f_{m}, f_{0}-G_{m}\right\rangle=\left\langle f_{m}, r_{m}+\sum_{j=0}^{m-1} c_{k_{j}} \widetilde{g}_{k_{j}}-\sum_{j=0}^{m-1}\left\langle f_{j}, g_{j+1}\right\rangle g_{j+1}\right\rangle \\
& \leqslant\left|\left\langle f_{m}, r_{m}\right\rangle\right|+\sum_{j=0}^{m-1}\left|c_{k_{j}}\right|\left|\left\langle f_{m}, \widetilde{g}_{k_{j}}\right\rangle\right|+\sum_{j=0}^{m-1}\left|\left\langle f_{j}, g_{j+1}\right\rangle\right|\left|\left\langle f_{m}, g_{j+1}\right\rangle\right| \\
& \leqslant\left|\left\langle f_{m}, r_{m}\right\rangle\right|+\sum_{j=0}^{m-1}\left(\left|c_{k_{j}}\right|+\left|\left\langle f_{j}, g_{j+1}\right\rangle\right|\right)\left|\left\langle f_{m}, g_{m+1}\right\rangle\right| \\
& \leqslant\left\|f_{m}\right\|\left\|r_{m}\right\|+d_{m} \sum_{j=0}^{m-1}\left(d_{j}+\left|c_{k_{j}}\right|\right) \leqslant a_{m}^{1 / 2} m^{-\alpha}+d_{m}\left(b_{m}+m^{\beta}\right) \\
& d_{m} \geqslant \frac{a_{m}-a_{m}^{1 / 2} m^{-\alpha}}{b_{m}+m^{\beta}}
\end{aligned}
$$


Пусть

$$
\varepsilon=\min \left(\varepsilon_{0}, \frac{1}{2}-\alpha-\beta, \alpha\right) .
$$

Рассмотрим числа $m_{0} \in \mathbb{N}$ со следуюшим свойством:

$$
a_{m_{0}} \geqslant m_{0}^{-2 \alpha+\varepsilon / 5}, \quad a_{m_{0}-1}<\left(m_{0}-1\right)^{-2 \alpha+\varepsilon / 5},
$$

и промежуток натурального ряда $\Delta\left(m_{0}\right)$, равный

$$
\Delta\left(m_{0}\right)=\left\{m_{0}, m_{0}+1, \ldots, s-1\right\},
$$

где $s$ - минимальное число, большее $m_{0}$, для которого $a_{s}<s^{-2 \alpha+\varepsilon / 5}$, или, если такого $s$ не существует, равньй

$$
\Delta\left(m_{0}\right)=\left\{m_{0}, m_{0}+1, \ldots\right\} .
$$

Таким образом, для всех $k \in \Delta\left(m_{0}\right)$ выполняется неравенство

$$
a_{k} \geqslant k^{-2 \alpha+\varepsilon / 5} \text {. }
$$

Ясно, что для доказательства теоремы достаточно проверить, что для всех $m_{0}$, удовлетворяющих $(2.3)$, и $m \in \Delta\left(m_{0}\right)$ вьполняется

$$
a_{m}=\left\|f_{m}\right\|^{2} \leqslant m^{-2 \alpha /(1+2 \alpha)+2 \varepsilon} .
$$

Мы возьмем настолько малое $\delta$, что первое $m_{0}$, удовлетворяющее (2.3), будет достаточно большим (настолько, насколько это необходимо для верности последующих выкладок). В частности, будем требовать выполнения неравенства

$$
a_{m_{0}}<a_{m_{0}-1} \leqslant\left(m_{0}-1\right)^{-2 \alpha+\varepsilon / 5}<m_{0}^{-2 \alpha+\varepsilon / 4} .
$$

В зависимости от значения $b_{m_{0}}$ рассмотрим два случая.

Случай 1: $b_{m_{0}} \geqslant m_{0}^{1 / 2-\alpha-\varepsilon / 10}$.

ЛЕмма 2.1. Если $b_{m_{0}} \geqslant m_{0}^{1 / 2-\alpha-\varepsilon / 10}$, то для всех $m \in \Delta\left(m_{0}\right)$ выполняется

$$
b_{m} \geqslant m^{\beta+\varepsilon / 10} \text {. }
$$

ДоКАЗАТЕльСтво. Предположим противное. Тогда рассмотрим минимальное $m \in$ $\Delta\left(m_{0}\right)$, для которого

$$
b_{m}<m^{\beta+\varepsilon / 10} .
$$

Пусть $m_{0} \leqslant k \leqslant m-1$, тогда

$$
b_{k} \geqslant k^{\beta+\varepsilon / 10} .
$$

В силу монотонности последовательностей $\left\{a_{k}\right\},\left\{b_{k}\right\}$, а также соотношений $(2.1),(2.4)$ и (2.7) вьполняются следующие неравенства:

$$
d_{k} \geqslant \frac{a_{k}-a_{k}^{1 / 2} k^{-\alpha}}{b_{k}+k^{\beta}}=\frac{a_{k}\left(1-k^{-\alpha} / a_{k}^{1 / 2}\right)}{b_{k}\left(1+k^{\beta} / b^{k}\right)} \geqslant \frac{m^{-2 \alpha+\varepsilon / 5}}{m^{\beta+\varepsilon / 10}} \frac{\left(1-k^{-\alpha} / a_{k}^{1 / 2}\right)}{\left(1+k^{\beta} / b^{k}\right)} .
$$


Отсюда в силу (2.4) и (2.8) для достаточно больших $k$ и $m$ получаем

$$
d_{k} \geqslant \frac{m^{-2 \alpha+\varepsilon / 5}}{m^{\beta+\varepsilon / 8}}
$$

Поэтому

$$
b_{m}=b_{m_{0}}+\sum_{k=m_{0}}^{m-1} d_{k} \geqslant m_{0}^{1 / 2-\alpha-\varepsilon / 10}+\frac{m^{-2 \alpha+\varepsilon / 5}}{m^{\beta+\varepsilon / 8}}\left(m-m_{0}\right) .
$$

Проверим, что

$$
b_{m} \geqslant m^{\beta+\varepsilon / 10} \text {. }
$$

Для доказательства (2.9) рассмотрим функцию

$$
\Phi(t)=m_{0}^{1 / 2-\alpha-\varepsilon / 10}+\frac{t^{-2 \alpha+\varepsilon / 5}}{t^{\beta+\varepsilon / 8}}\left(t-m_{0}\right)-t^{\beta+\varepsilon / 10} .
$$

Достаточно показать, что $\Phi(t) \geqslant 0$. Проверим, что

$$
\Phi\left(m_{0}\right) \geqslant 0, \quad \Phi^{\prime}(t) \geqslant 0 \text { при } t \geqslant m_{0} .
$$

Действительно, для достаточно большого $m_{0}$

$$
\begin{aligned}
\Phi^{\prime}(t)= & (1-2 \alpha-\beta+0.075 \varepsilon) t^{-2 \alpha-\beta+0.075 \varepsilon} \\
& -(-2 \alpha-\beta+0.075 \varepsilon) t^{-2 \alpha-\beta+0.075 \varepsilon-1} m_{0}-(\beta+\varepsilon / 10) t^{\beta+\varepsilon / 10-1} \\
\geqslant & t^{-2 \alpha-\beta+0.075 \varepsilon}-(\beta+\varepsilon / 10) t^{\beta+\varepsilon / 10-1} \geqslant 0 .
\end{aligned}
$$

В последнем неравенстве была использована оценка $-2 \alpha-\beta+0.075 \varepsilon>\beta-1+\varepsilon / 10$, которая вытекает из соотношения (2.2). Для завершения доказательства леммы оценим $\Phi\left(m_{0}\right)$ :

$$
\Phi\left(m_{0}\right)=m_{0}^{1 / 2-\alpha-\varepsilon / 10}-(\beta+\varepsilon / 10) m_{0}^{\beta+\varepsilon / 10}>0 .
$$

В случае 1 лемма 2.1 и соотношение (2.4) позволяют преобразовать (2.1) для $m \in$ $\Delta\left(m_{0}\right)$ :

$$
d_{m} \geqslant \frac{a_{m}-a_{m}^{1 / 2} m^{-\alpha}}{b^{m}+m^{\beta}} \geqslant \frac{a_{m}\left(1-m^{-\varepsilon / 10}\right)}{b_{m}\left(1+m^{-\varepsilon / 10}\right)} \geqslant \frac{a_{m}}{b_{m}\left(1+m^{-\varepsilon / 20}\right)} .
$$

Пусть $m \in \Delta\left(m_{0}\right)$. Тогда из определения ЧЖА вытекает

$$
a_{m+1}=a_{m}-d_{m}^{2}=a_{m}\left(1-\frac{d_{m}^{2}}{a_{m}}\right) \text {. }
$$

С другой стороны, используя (2.10), получим

$$
b_{m+1}=b_{m}+d_{m}=b_{m}\left(1+\frac{d_{m}^{2}}{d_{m} b_{m}}\right) \leqslant b_{m}\left(1+\frac{d_{m}^{2}}{a_{m}}\left(1+m^{-\varepsilon / 20}\right)\right) .
$$

Из двух последних соотношений вытекает следующее неравенство:

$$
a_{m+1} b_{m+1} \leqslant a_{m} b_{m}\left(1+\frac{d_{m}^{2}}{a_{m}} m^{-\varepsilon / 20}\right) .
$$

Тогда

$$
a_{m} b_{m} \leqslant a_{m_{0}} b_{m_{0}} \prod_{k=m_{0}}^{m-1}\left(1+\frac{d_{k}^{2}}{a_{k}} k^{-\varepsilon / 20}\right)
$$


Лемма 2.2. Существует такая константа $C=C(\alpha, \varepsilon)>0$, что для произвольных $m_{0}$ и $m$, удовлетворяющ, их неравенствам $m_{0}<m<m_{0}^{2 /(1-6 \alpha)}, a_{m} \geqslant m^{-2 \varepsilon+\alpha / 5}$ и (2.12), имеет место следующая оченка:

$$
a_{m} b_{m} \leqslant C a_{m_{0}} b_{m_{0}} .
$$

ДоКАЗАТЕЛЬСтво. Из (2.11) вытекает, что

$$
a_{m}=a_{m_{0}} \prod_{k=m_{0}}^{m-1}\left(1-\frac{d_{k}^{2}}{a_{k}}\right)
$$

Принимая во внимание то, что $a_{m_{0}}<1, m<m_{0}^{2 /(1-6 \alpha)}$ и $a_{m} \geqslant m^{-2 \varepsilon+\alpha / 5}$, получим

$$
\begin{gathered}
\left(m_{0}^{2 /(1-6 \alpha)}\right)^{-2 \alpha+\varepsilon / 5} \leqslant \prod_{k=m_{0}}^{m-1}\left(1-\frac{d_{k}^{2}}{a_{k}}\right), \\
\frac{2}{1-6 \alpha}\left(-2 \alpha+\frac{\varepsilon}{5}\right) \ln m_{0} \leqslant \sum_{k=m_{0}}^{m-1} \ln \left(1-\frac{d_{k}^{2}}{a_{k}}\right), \quad \sum_{k=m_{0}}^{m-1} \frac{d_{k}^{2}}{a_{k}} \leqslant \frac{2}{1-6 \alpha}\left(2 \alpha-\frac{\varepsilon}{5}\right) \ln m_{0} .
\end{gathered}
$$

Ясно, что найдется такая константа $C>0$, что для всех $m_{0}$ выполняется

$$
\sum_{k=m_{0}}^{m-1} \frac{d_{k}^{2}}{a_{k}} k^{-\varepsilon / 20} \leqslant \sum_{k=m_{0}}^{m-1} \frac{d_{k}^{2}}{a_{k}} m_{0}^{-\varepsilon / 20} \leqslant m_{0}^{-\varepsilon / 20} \frac{2}{1-6 \alpha}\left(2 \alpha-\frac{\varepsilon}{5}\right) \ln m_{0} \leqslant \ln C .
$$

Тогда, используя (2.12), получим

$$
\begin{aligned}
a_{m} b_{m} & \leqslant a_{m_{0}} b_{m_{0}} \exp \left(\sum_{k=m_{0}}^{m-1} \ln \left(1+\frac{d_{k}^{2}}{a_{k}} k^{-\varepsilon / 20}\right)\right) \\
& \leqslant a_{m_{0}} b_{m_{0}} \exp \left(\sum_{k=m_{0}}^{m-1} \frac{d_{k}^{2}}{a_{k}} k^{-\varepsilon / 20}\right) \leqslant C a_{m_{0}} b_{m_{0}}
\end{aligned}
$$

что доказьвает (2.13).

Из леммы 2.2 непосредственно вытекает

СледСтвиЕ 2.1. Существует такая константа $C>0$, что в случае 1 для всех $m_{0}$, обладающих свойством (2.3), и $m \in \Delta\left(m_{0}\right), m<m_{0}^{2 /(1-6 \alpha)}$, выполняется неравенство

$$
a_{m} b_{m} \leqslant C a_{m_{0}} b_{m_{0}}
$$

Пусть

$$
n_{0}=\left[m_{0}^{1+2 \alpha}\right]+1 .
$$

Тогда справедлива следующая 
ЛЕмма 2.3. Пусть $m_{0}$ обладает свойством (2.3) и $n_{0}$ определено соотношением (2.14). Тогда для всех $m \in \Delta\left(m_{0}\right), m \geqslant n_{0}$, выполняется неравенство

$$
\frac{a_{m}}{b_{m}^{2}} \leqslant \frac{2}{m}
$$

ДоКАЗАТЕЛьСТво. Сначала проверим (2.15) для $m=n_{0}$. Ясно, что

$$
b_{n_{0}} \geqslant \sum_{k=m_{0}}^{n_{0}-1} d_{k}
$$

Так как $m_{0}$ достаточно велико, то, используя $(2.10)$, получим

$$
b_{n_{0}} \geqslant\left(n_{0}-m_{0}\right) \frac{a_{n_{0}}}{1.5 b_{n_{0}}}, \quad b_{n_{0}} \geqslant n_{0} \frac{a_{n_{0}}}{2 b_{n_{0}}}, \quad \frac{a_{n_{0}}}{b_{n_{0}}^{2}} \leqslant \frac{2}{n_{0}} .
$$

С другой стороны, следуя работе [2] и используя (2.10) и (2.11), получаем

$$
\frac{a_{m+1}}{b_{m+1}^{2}} \leqslant \frac{a_{m+1}}{b_{m}^{2}}=\frac{a_{m}}{b_{m}^{2}}\left(1-\frac{d_{m}^{2}}{a_{m}}\right) \leqslant \frac{a_{m}}{b_{m}^{2}}\left(1-\frac{a_{m}}{2 b_{m}^{2}}\right) .
$$

Из этого неравенства, (2.16) и леммы 3.4 из работы [2] (точнее, из индукционного перехода из этой леммы) непосредственно следует справедливость (2.15) для $m \geqslant n_{0}$.

ДОКАЗАТЕЛЬСТВо ТЕОРЕМЫ 1 В СЛУчАЕ 1 . Сначала Проверим $(2.5)$ для $m, m_{0} \leqslant$ $m \leqslant n_{0}, m \in \Delta\left(m_{0}\right)$. Из $(2.6)$ и $(2.14)$ вытекает

$$
a_{m} \leqslant a_{m_{0}} \leqslant m_{0}^{-2 \alpha+\varepsilon / 4} \leqslant\left((m-1)^{1 /(1+2 \alpha)}\right)^{-2 \alpha+\varepsilon / 4} \leqslant m^{-2 \alpha /(1+2 \alpha)+2 \varepsilon} .
$$

Теперь проверим (2.5) для $m, n_{0}<m \leqslant m_{0}^{2 /(1-6 \alpha)}, m \in \Delta\left(m_{0}\right)$. Оценим $a_{m_{0}} b_{m_{0}}$. Начнем с $b_{m_{0}}: b_{m_{0}}=\sum_{k=0}^{m_{0}-1} d_{k}$, но

$$
\sum_{k=0}^{m-1} d_{k}^{2} \leqslant a_{0} \leqslant 1
$$

Тогда по неравенству Коши-Буняковского

$$
b_{m_{0}} \leqslant m_{0}^{1 / 2}
$$

Учитьвая (2.6), получаем

$$
a_{m_{0}} b_{m_{0}} \leqslant m_{0}^{-2 \alpha+\varepsilon / 4+1 / 2}
$$

По следствию 2.1

$$
a_{m} b_{m} \leqslant C a_{m_{0}} b_{m_{0}} \leqslant C m_{0}^{-2 \alpha+\varepsilon / 4+1 / 2}
$$

по лемме 2.3

$$
\frac{a_{m}}{b_{m}^{2}} \leqslant \frac{2}{m}
$$


Следовательно, для больших $m_{0}$ имеем

$$
\begin{gathered}
a_{m}^{3}=\left(a_{m} b_{m}\right)^{2} \frac{a_{m}}{b_{m}^{2}} \leqslant \frac{m_{0}^{-4 \alpha+\varepsilon / 2+1}}{m} 2 C^{2} \leqslant \frac{m_{0}^{-4 \alpha+\varepsilon+1}}{m}, \\
a_{m} \leqslant \frac{m_{0}^{(-4 \alpha+\varepsilon+1) / 3}}{m^{1 / 3}}, \\
a_{m} \leqslant \frac{m^{1 /(1+2 \alpha)^{(-4 \alpha+\varepsilon+1) / 3}}}{m^{1 / 3}} \leqslant m^{(-6 \alpha+\varepsilon) /(3(1+2 \alpha))} \leqslant m^{-2 \alpha /(1+2 \alpha)+2 \varepsilon} .
\end{gathered}
$$

Заметим, что из $(2.18)$ вытекает, что при $l=\left[m_{0}^{2 /(1-6 \alpha)}\right]$ вьполняется

$$
a_{l} \leqslant l^{-2 \alpha}<l^{-2 \alpha+\varepsilon / 5} \text {. }
$$

Действительно,

$$
a_{l} \leqslant \frac{m_{0}^{(-4 \alpha+\varepsilon / 2+1) / 3}}{l^{1 / 3}} \leqslant \frac{m_{0}^{1 / 2}}{\left(m_{0}-1\right)^{2 /(3(1-6 \alpha))}} \leqslant m_{0}^{-4 \alpha /(1-6 \alpha)} \leqslant l^{-2 \alpha}
$$

здесь мы воспользовались тем, что

$$
\frac{1}{2}<\frac{2}{3}=\frac{2 / 3-4 \alpha}{1-6 \alpha}
$$

Поэтому $l=\left[m_{0}^{2 /(1-6 \alpha)}\right] \notin \Delta\left(m_{0}\right)$ и, следовательно, последний случай $m \in \Delta\left(m_{0}\right)$ : $m \geqslant m_{0}^{2 /(1-6 \alpha)}$ не нуждается в рассмотрении (таких $m$ просто нет). Случай 1 полностью рассмотрен.

Случай $2: b_{m_{0}}<m_{0}^{1 / 2-\alpha-\varepsilon / 10}$.

ДокаЗАТЕЛЬСТво ТЕОРЕмЫ 1 в СЛУчаЕ 2 . Докажем, что в случае 2 промежуток $\Delta\left(m_{0}\right)=\left\{m_{0}\right\}$, другими словами,

$$
a_{m_{0}+1}<\left(m_{0}+1\right)^{-2 \alpha+\varepsilon / 5}
$$

Из (2.3) следует, что

$$
m_{0}^{-2 \alpha+\varepsilon / 5} \leqslant a_{m_{0}}<a_{m_{0}-1} \leqslant\left(m_{0}-1\right)^{-2 \alpha+\varepsilon / 5} .
$$

Поэтому для доказательства (2.19) достаточно проверить, что

$$
d_{m_{0}}^{2} \geqslant\left(m_{0}-1\right)^{-2 \alpha+\varepsilon / 5}-\left(m_{0}+1\right)^{-2 \alpha+\varepsilon / 5} .
$$

Проверим, что

$$
d_{m_{0}} \geqslant 2 m_{0}^{-1 / 2-\alpha+\varepsilon / 10} .
$$

Действительно, из (2.1) и неравенств

$$
m^{\beta} \leqslant m^{1 / 2-\alpha-\varepsilon}, \quad a_{m_{0}} \leqslant m_{0}^{-2 \alpha+\varepsilon / 5}
$$

вытекает, что для достаточно больших $m_{0}$ вьполняется неравенство

$$
d_{m_{0}} \geqslant \frac{m_{0}^{-2 \alpha+\varepsilon / 5}}{m_{0}^{1 / 2-\alpha}}=m_{0}^{-1 / 2-\alpha+\varepsilon / 5}>2 m_{0}^{-1 / 2-\alpha+\varepsilon / 10} .
$$

Таким образом, теорема 1 полностью доказана. 
3. Доказательство теоремы 2. Так же, как и при доказательстве теоремы 1 , нам достаточно проверить справедливость следующего утверждения: для любого $\varepsilon_{0}>0$ существует такое $\delta>0$, что для всех функций $f \in A_{\alpha, \beta}^{1,1}(\mathscr{D}),\|f\|<\delta<1$, вьполняется

$$
\left\|f-G_{m}(f, D)\right\| \leqslant m^{-(1 / 2-\beta)+\varepsilon_{0}} .
$$

Будем действовать в обозначениях п. 2. Пусть

$$
\varepsilon=\min \left(\varepsilon_{0}, \alpha+\beta-\frac{1}{2}, \alpha, \frac{\beta}{10}, \frac{1}{2}-\beta\right) .
$$

Мы докажем, что если для $m_{1}:=m$ выполняются неравенства

$$
\begin{aligned}
a_{m_{1}} & >m_{1}-(1-2 \beta)+\varepsilon / 2 \\
a_{m_{1}-1} & \leqslant\left(m_{1}-1\right)^{-(1-2 \beta)+\varepsilon / 2}
\end{aligned}
$$

то уже для $m_{1}:=m+1$ неравенство (3.3) неверно. Таким образом, для достаточно больших $m$ будет вьполняться

$$
\begin{gathered}
\text { или } a_{m} \leqslant m^{-(1-2 \beta)+\varepsilon / 2}, \quad \text { или } a_{m}<a_{m-1} \leqslant(m-1)^{-(1-2 \beta)+\varepsilon / 2}, \\
a_{m} \leqslant m^{-(1-2 \beta)+0.51 \varepsilon} .
\end{gathered}
$$

Выберем $\delta$ таким образом, что для всех $1 \leqslant n \leqslant n_{0}$

$$
a_{n} \leqslant \delta \leqslant n_{0}^{-2 \alpha} \leqslant n^{-2 \alpha} \leqslant n^{-(1-2 \beta)}
$$

где $n_{0}$ - наперед заданное число. Это обеспечивает справедливость (3.5) для малых $m$ и дает вьполнение (3.1).

Пусть для $m_{1}$ выполняются (3.3) и (3.4) и для всех $m \leqslant m_{1}$ выполняется (3.5). Проверим, что

$$
a_{m_{1}+1} \leqslant\left(m_{1}+1\right)^{-(1-2 \beta)+\varepsilon / 2} .
$$

В зависимости от значения $b_{m_{1}}$ рассмотрим два случая.

Случай 1: $b_{m_{1}} \leqslant m_{1}^{\beta+\varepsilon / 5}$.

Из неравенств $(2.1),(3.3),(3.5)$ и $\varepsilon \leqslant \alpha+\beta-1 / 2$ (см. (3.2)) следует, что для достаточно больших $m_{1}$

$$
\begin{aligned}
d_{m_{1}} & \geqslant \frac{a_{m_{1}}-a_{m_{1}}^{1 / 2} m_{1}-\alpha}{b_{m_{1}}+m_{1} \beta} \geqslant \frac{m_{1}^{-(1-2 \beta)+\varepsilon / 2}-m_{1}^{1 / 2(-(1-2 \beta)+0.51 \varepsilon)} m_{1}^{-\alpha}}{m_{1}^{\beta+\varepsilon / 5}+m_{1}^{\beta}} \\
& =\frac{m_{1}^{(-(1-2 \beta)+\varepsilon / 2)}\left(1-m_{1}^{1 / 2-\alpha-\beta-0.245 \varepsilon}\right)}{m_{1}^{\beta+\varepsilon / 5}\left(1+m_{1}^{-\varepsilon / 5}\right)} \geqslant m_{1}^{-1-\beta+0.28 \varepsilon}, \\
d_{m_{1}}^{2} & =m_{1}^{-2+2 \beta+0.56 \varepsilon}>10 m_{1}^{-(1-2 \beta)+\varepsilon / 2-1} .
\end{aligned}
$$

Согласно (2.11) и (3.4)

$$
\begin{aligned}
a_{m_{1}+1} & =a_{m_{1}}-d_{m_{1}}^{2}<a_{m_{1}-1}-d_{m_{1}}^{2} \\
& \leqslant\left(m_{1}-1\right)^{-(1-2 \beta)+\varepsilon / 2}-10 m_{1}^{-(1-2 \beta)+\varepsilon / 2-1}<\left(m_{1}+1\right)^{-(1-2 \beta)+\varepsilon / 2}
\end{aligned}
$$


что и доказьвает (3.7) в случае 1.

Случай $2: b_{m_{1}}>m_{1}^{\beta+\varepsilon / 5}$.

Покажем, что этот случай вообще не может реализоваться. Предположим противное. Пусть $m_{0}$ - наибольшее из чисел, не превосходящих $m_{1}$, для которых вьполняется хотя бы одно из следующих утверждений:

$$
\begin{gathered}
m_{0}-1 \leqslant m_{1}^{(1-6 \alpha) / 2}, \\
b_{m_{0}-1} \leqslant\left(m_{0}-1\right)^{\beta+\varepsilon / 10} \\
a_{m_{0}-1} \leqslant\left(m_{0}-1\right)^{-2 \alpha+\varepsilon / 5} .
\end{gathered}
$$

В силу неравенств (3.6) такое $m_{0}$ найдется. Следовательно, для $m, m_{0} \leqslant m \leqslant m_{1}$, выполняются неравенства

$$
\begin{gathered}
b_{m}>m^{\beta+\varepsilon / 10}, \\
a_{m} \geqslant m^{-2 \alpha+\varepsilon / 5}, \\
m_{0}^{2 /(1-6 \alpha)} \geqslant m_{1} .
\end{gathered}
$$

Из (2.1), (3.11) и (3.12) вытекает, что для $m, m_{0} \leqslant m \leqslant m_{1}$, справедлива оценка $(2.10)$ :

$$
d_{m} \geqslant \frac{a_{m}-a_{m}^{1 / 2} m^{-\alpha}}{b^{m}+m^{\beta}} \geqslant \frac{a_{m}\left(1-m^{-\varepsilon / 10}\right)}{b_{m}\left(1+m^{-\varepsilon / 10}\right)} \geqslant \frac{a_{m}}{b_{m}\left(1+m^{-\varepsilon / 20}\right)} .
$$

Отсюда следует (см. п. 2) неравенство (2.12):

$$
a_{m} b_{m} \leqslant a_{m_{0}} b_{m_{0}} \prod_{k=m_{0}}^{m-1}\left(1+\frac{d_{k}^{2}}{a_{k}} k^{-\varepsilon / 20}\right)
$$

Тогда с учетом (3.13) мы можем применить лемму 2.2 при $m=m_{1}$ и, используя (3.3), получим

$$
\begin{gathered}
a_{m_{1}} b_{m_{1}} \leqslant C a_{m_{0}} b_{m_{0}} \\
m_{1}^{-1+3 \beta+0.7 \varepsilon}=m_{1}^{-(1-2 \beta)+\varepsilon / 2} m_{1}^{\beta+\varepsilon / 5} \leqslant a_{m_{1}} b_{m_{1}} \leqslant C a_{m_{0}} b_{m_{0}} .
\end{gathered}
$$

Заметим также, что из неравенств

$$
\frac{\alpha}{1+2 \alpha}+\beta \geqslant \frac{1}{2}, \quad \alpha<\frac{1}{6}, \quad \beta<\frac{1}{2}
$$

вытекают оценки

$$
\begin{gathered}
\frac{1}{2}>\beta \geqslant \frac{1 / 2}{1+2 \alpha} \geqslant \frac{3}{8}, \\
\alpha \geqslant \frac{1}{4 \beta}-\frac{1}{2} .
\end{gathered}
$$


Предположим, что для $m_{0}$ вьполняется (3.8). Тогда согласно $(2.17),(3.5)$ и (3.14) должно выполняться следующее неравенство:

$$
m_{1}^{-1+3 \beta+0.7 \varepsilon} \leqslant C a_{m_{0}} b_{m_{0}} \leqslant C m_{0}^{-(1-2 \beta)+0.51 \varepsilon} m_{0}^{1 / 2} .
$$

Соотношения (3.8) и (3.16) дают

$$
m_{1} \geqslant\left(m_{0}-1\right)^{2 /(1-6 \alpha)}>m_{0}^{1.5 /(1-6 \alpha)} \geqslant m_{0}^{1.5 /(1-6(1 /(4 \beta)-1 / 2))}=m_{0}^{3 \beta /(8 \beta-3)} .
$$

Подставляя эту оценку в предыдущее неравенство и учитьвая, что $\varepsilon \leqslant \beta / 10$ (см. (3.2)), получаем

$$
\begin{gathered}
m_{0}^{3 \beta(-1+3 \beta+0.7 \varepsilon) /(8 \beta-3)} \leqslant C m_{0}^{2 \beta-1 / 2+0.51 \varepsilon}, \\
m_{0}^{3 \beta(-1+3 \beta+0.7 \varepsilon) /(8 \beta-3)-(2 \beta-1 / 2+0.51 \varepsilon)} \leqslant C, \\
m_{0}^{3 \beta(-1+3 \beta) /(8 \beta-3)-(2 \beta-1 / 2+\beta / 10)} \leqslant C .
\end{gathered}
$$

Но это неравенство не может выполняться в силу того, что

$$
\frac{3 \beta(-1+3 \beta)}{8 \beta-3}-\left(2 \beta-\frac{1}{2}+\frac{\beta}{10}\right)>0.001
$$

при $3 / 8 \leqslant \beta \leqslant 1 / 2$. (Последняя оценка может быть получена непосредственной проверкой.) Значит, $m_{0}$ не может удовлетворять (3.8).

Предположим, что $m_{0}$ удовлетворяет (3.9). Тогда

$$
b_{m_{0}}=b_{m_{0}-1}+d_{m_{0}-1} \leqslant\left(m_{0}-1\right)^{\beta+\varepsilon / 10}+a_{m_{0}-1}^{1 / 2}<m_{0}^{\beta+0.11 \varepsilon} .
$$

Согласно (3.5) и (3.14)

$$
m_{1}^{-1+3 \beta+0.7 \varepsilon} \leqslant C m_{0}^{(\beta+0.11 \varepsilon)+(-(1-2 \beta)+0.51 \varepsilon)}
$$

что не может быть верно, так как $m_{1} \geqslant m_{0}$ и $-1+3 \beta+0.7 \varepsilon>-1+3 \beta+0.62 \varepsilon>0$. Поэтому $m_{0}$ не может удовлетворять (3.9).

Рассмотрим последнюю возможность: $m_{0}$ удовлетворяет (3.10). В силу того, что $a_{m_{1}}<a_{m_{0}-1}$ и $-(1-2 \beta)+\varepsilon / 2<0($ см. $(3.2))$, с учетом $(3.3)$ и $(3.10)$ имеем

$$
\begin{aligned}
m_{1}^{-(1-2 \beta)+\varepsilon / 2} & <a_{m_{1}}<a_{m_{0}-1} \leqslant\left(m_{0}-1\right)^{-2 \alpha+\varepsilon / 5} \\
m_{1} & >m_{0}^{(2 \alpha-0.19 \varepsilon) /(1-2 \beta-\varepsilon / 2)} .
\end{aligned}
$$

Соотношения $(2.17),(3.10),(3.14)$ и (3.17) дают

$$
\begin{gathered}
m_{1}^{-1+3 \beta+0.7 \varepsilon} \leqslant C a_{m_{0}} b_{m_{0}} \leqslant C a_{m_{0}-1} b_{m_{0}}, \\
m_{0}^{(2 \alpha-0.19 \varepsilon)(-1+3 \beta+0.7 \varepsilon) /(1-2 \beta-\varepsilon / 2)} \leqslant C\left(m_{0}-1\right)^{-2 \alpha+\varepsilon / 5} m_{0}^{1 / 2}<C m_{0}^{1 / 2-2 \alpha+0.21 \varepsilon} .
\end{gathered}
$$


Легко проверяется, что

$$
f(t)=\frac{-1+3 t+0.7 \varepsilon}{1-2 t-\varepsilon / 2}
$$

возрастает на отрезке [3/8,1/2- $\varepsilon / 2]$; поэтому, используя (3.15), получим

$$
\begin{gathered}
m_{0}^{\frac{(2 \alpha-0.19 \varepsilon)(-1+3 /(2(1+2 \alpha))+0.7 \varepsilon)}{1-1 /(1+2 \alpha)-\varepsilon / 2}} \leqslant m_{0}^{(2 \alpha-0.19 \varepsilon)(-1+3 \beta+0.7 \varepsilon) /(1-2 \beta-\varepsilon / 2)}, \\
\frac{(2 \alpha-0.19 \varepsilon)(-1+3 /(2(1+2 \alpha))+0.7 \varepsilon)}{1-1 /(1+2 \alpha)-\varepsilon / 2} \leqslant C\left(m_{0}-1\right)^{-2 \alpha+\varepsilon / 5} m_{0}^{1 / 2}<C m_{0}^{1 / 2-2 \alpha+0.21 \varepsilon}, \\
m_{0}^{\frac{(2 \alpha-0.19 \varepsilon)(1 / 2-2 \alpha+0.7(1+2 \alpha) \varepsilon)}{2 \alpha-0.5(1+2 \alpha) \varepsilon}} \leqslant C m_{0}^{1 / 2-2 \alpha+0.21 \varepsilon}, \\
m_{0}^{1 / 2-2 \alpha+0.7(1+2 \alpha) \varepsilon} \leqslant C m_{0}^{1 / 2-2 \alpha+0.21 \varepsilon},
\end{gathered}
$$

что не может быть верно для больших $m_{0}$ (показатель в левой части больше показателя в правой). Таким образом, $m_{0}$ не может удовлетворять (3.10), случай 2 невозможен, и теорема 2 полностью доказана.

4. Построение примера 1. Пусть $\left\{e_{n}\right\}_{n=0}^{\infty}$ - ортонормированньй базис в гильбертовом пространстве $H$. Рассмотрим векторы

$$
h_{0}=e_{0}, \quad h_{n}=e_{0}+n^{-S} e_{n}, \quad n \geqslant 1,
$$

где $S>\gamma+1 / 2$ будет определено позже. Определим словарь

$$
D=\left\{\frac{h_{n}}{\left\|h_{n}\right\|}\right\}_{n=0}^{\infty} .
$$

Положим

$$
f_{0}=\sum_{n=1}^{\infty} n^{-\gamma-1 / 2} e_{n}
$$

Легко видеть, что

$$
\sigma_{m}\left(f_{0}, \mathscr{D}\right) \leqslant\left(\sum_{n=m}^{\infty} n^{-2 \gamma-1}\right)^{1 / 2} \leqslant \frac{1}{(2 \gamma)^{1 / 2}}(m-1)^{-\gamma},
$$

откуда следует, что $f \in A^{\gamma}(\mathscr{D})$. Будем действовать в обозначениях, введенных в начале п. 2. Оценим величину $a_{0}$ :

$$
a_{0}=\left\langle f_{0}, f_{0}\right\rangle=\sum_{n=1}^{\infty} n^{-2 \gamma-1} \leqslant\left(1+\frac{1}{2 \gamma}\right) .
$$

Согласно неравенству Коши-Буняковского

$$
b_{m}=\sum_{n=0}^{m} d_{n} \leqslant m^{1 / 2}\left(\sum_{n=0}^{m} d_{n}^{2}\right)^{1 / 2} \leqslant m^{1 / 2} a_{0}^{1 / 2} \leqslant m^{1 / 2}\left(\frac{1}{2 \gamma}+1\right)^{1 / 2} .
$$


Учитьвая, что все векторы словаря $\mathscr{D}$ пропорциональны векторам $h_{n}, n \geqslant 0$, и то, что $\left\|h_{n}\right\| \geqslant 1, n \geqslant 0$, имеем

$$
f_{0}-f_{m}=\sum_{n=0}^{\infty} c_{n} h_{n}
$$

где лишш конечное число $(\leqslant m)$ коэффициентов $c_{n}$ отличны от нуля и

$$
\left|c_{n}\right| \leqslant m^{1 / 2}\left(\frac{1}{2 \gamma}+1\right)^{1 / 2}, \quad n \geqslant 0 .
$$

Следовательно,

$$
\left|\left\langle f_{0}-f_{m}, e_{n}\right\rangle\right|=\left|\left\langle c_{n} h_{n}, e_{n}\right\rangle\right| \leqslant m^{1 / 2}\left(\frac{1}{2 \gamma}+1\right)^{1 / 2} n^{-S}, \quad n \geqslant 1 .
$$

Обозначим

$$
\lambda=\frac{1 / 2}{S-\gamma-1 / 2} .
$$

Ясно, что найдется такое число $C_{1}$, что для $n>C_{1} m^{\lambda}$ выполняется

$$
m^{1 / 2}\left(\frac{1}{2 \gamma}+1\right)^{1 / 2} n^{-S}<\frac{1}{2} n^{-\gamma-1 / 2}
$$

Тогда, учитьвая (4.1) и (4.2), для $n>C_{1} m^{\lambda}$ имеем

$$
\left|\left\langle f_{m}, e_{n}\right\rangle\right| \geqslant\left|\left\langle f_{0}, e_{n}\right\rangle\right|-\left|\left\langle f_{0}-f_{m}, e_{n}\right\rangle\right| \geqslant \frac{1}{2} n^{-\gamma-1 / 2} .
$$

Отсюда

$$
\left\|f_{m}\right\|^{2} \geqslant \sum_{n=\left[C_{1} m^{\lambda}\right]+1}^{\infty}\left|\left\langle f_{m}, e_{n}\right\rangle\right|^{2} \geqslant \sum_{n=\left[C_{1} m^{\lambda}\right]+1}^{\infty} \frac{1}{4} n^{-2 \gamma-1} \geqslant C^{2} m^{-2 \lambda \gamma}
$$

что дает требуемую оценку

$$
\left\|f-G_{m}(f, \mathscr{D})\right\|=\left\|f_{m}\right\|>C m^{-\delta}
$$

В случае, если $\lambda<\delta / \gamma$, это верно при любом $S>2 \gamma / \delta+\gamma+1 / 2$.

\section{СПИСОК ЦИТИРОВАННОЙ ЛИТЕРАТУРЫ}

[1] Jones L. K On a conjecture of Huber concerning the convergence of PP-regression // Ann. Statist. 1987. V. 15. P. 880-882.

[2] DeVore R. A., Temlyakov V. N. Some remarks on greedy algorithms // Adv. Comput. Math. 1996. V. 5. P. 173-187.

[3] Konyagin S. V., Temlyakov V. N. Rate of convergence of pure greedy algorithms // East J. Approx. 1999. V. 5. P. 493-499.

[4] Livshitz E. D., Temlyakov V. N. Two lower estimates in greedy approximation // Constructive Approximation. 2003. V. 19. P. 509-524. 